more direct in his vision for the future of 454 sequencing. "My goal is simple, I want to displace Sanger sequencing for de novo sequencing."

\section{Counting games}

Like the Genome Sequencer FLX system, both Illumina and Applied Biosystems have used emulsion PCR as a starting point for their next-generation sequencing systems. But from there the methods of sequencing are quite different from each other.

In January this year, Illumina, located in San Diego, California, acquired the Haywardbased firm Solexa. Solexa's key technology, previously called the Solexa $1 \mathrm{G}$ and now named the Genome Analyzer, is a next-generation sequencing system that can sequence the equivalent of a third of the entire human genome in a single run. The Broad Institute now uses 16 Genome Analyzers for various projects. "Any application that is counting-related is a very good one to perform using Illumina's system," says Nusbaum.

Nusbaum and his colleagues, along with other groups, have already demonstrated the usefulness of the Genome Analyzer in

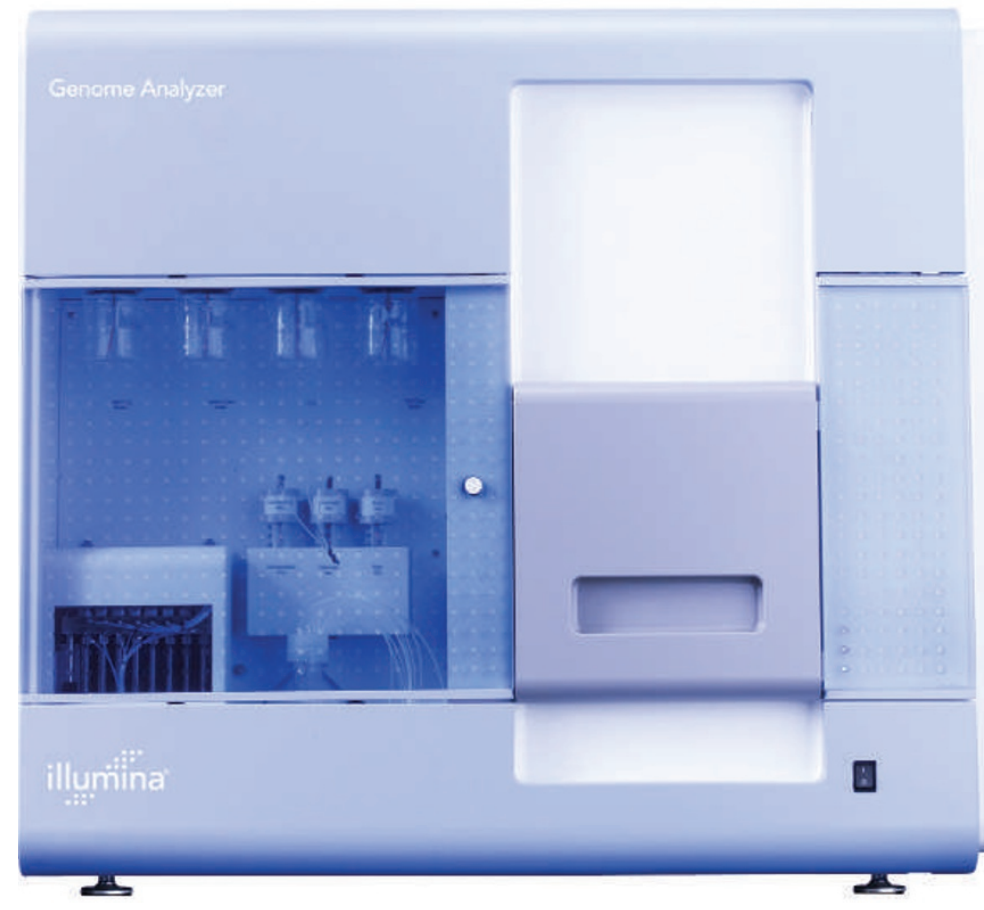

The Illumina Genome Analyzer has been used for several CHIP-sequencing projects.

and throughout develop- $₫$ ment. Nusbaum adds that it is also an incredibly easy application of the technology and anticipates that Illumina instruments will be used for other applications such as transcriptional profiling or microRNA and small RNA discovery. "It is also a great way to identify polymorphisms in genomes that are not extremely different."

Applied Biosystems in Foster City, California, is rolling out its new sequencing by oligonucleotide ligation and detection, or SOLiD, system in October 2007. The target is to cover a whole human genome in one run, says Kevin McKernan, senior director of scientific operations at Applied Biosystems. McKernan says that in-house, the SOLiD system has been obtaining around a giga-

looking at patterns of chromatin structure by using chromatin immunoprecipitation ${ }^{2-5}$. "It is an incredibly powerful application of the technology," says Nusbaum. By pulling down DNA bound to histones carrying specific modifications, sequencing it and mapping it back to the genome, they could map the status of chromatin across the genome base more data than their target, achieving 4 gigabases of sequence per run that aligns to the target genome, and 8 gigabases overall. McKernan thinks that with advances in the PCR process over time, this will turn into 8 gigabases of sequence that aligns to the target genome. The SOLiD system differs from other next-generation sequencing systems

\title{
CHIPPING OUT OUR DIFFERENCES
}

Single nucleotide polymorphism (SNP) genotyping is a method for determining genetic variation. As more and more SNPs have been identified from the genome in recent years, the power of this technique has steadily increased. Affymetrix, located in Santa Clara, California, is one company that is taking advantage of SNPdiscovery projects, such as the International Hap Map effort, to generate SNP arrays for wholegenome association studies. In May, Affymetrix launched its next-generation array, called the Genome-wide Human SNP Array 6.0, or SNP6.0. "This chip allows us to look simultaneously at more than 1.8 million markers of genetic variation," says Keith Jones, vice-president of assay and application product development at the company.

The SNP6.0 not only offers genome-wide SNP coverage, but also contains more than 900,000 probes that target copy-number variants (CNVs) in the genome. "When walking down the path in designing the SNP6.0, we took the biochemistry that we used to generate targets to hybridize to the arrays and empirically identified probes that responded in a dosedependent manner to changes in copy number," says Jones.

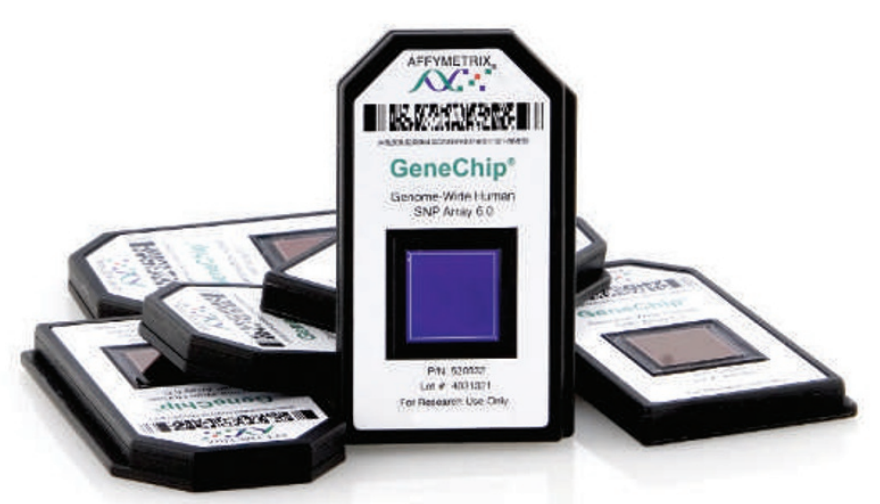

Affymetrix now offers the Genome-wide Human SNP Array 6.0 containing more than 1.8 million markers of genetic variation.
Other companies have also placed CNV probes onto their genotyping chips. Illumina, based in San Diego, California, now offers the Human 1M BeadChip, which boasts more than one million SNP and CNV probes, for whole-genome genotyping applications. Nimblegen, located in Madison, Wisconsin, and recently acquired by Roche Applied Sciences, also offers several whole-genome and custom-tiling array comparative genome hybridization products for examining copy-number variation across the entire genome. These arrays contain more than 385,000 probes at a median spacing of 6,000 base pairs. Agilent Technologies, located in Santa Clara, California, provides several array comparative genome hybridization products for analysis of copy-number variation in humans, mice and rats.

Illumina also offers several more-focused SNP arrays, including a cancer panel and one that targets the major histocompatibility complex, an area that Affymetrix also seems to be moving into. "I think it is also fair to say that you will see more application-specific SNP panels in the future," says Jones. 